\title{
НОВОЕ ГРАНИЧНОЕ УСЛОВИЕ ПРИ УСТАНОВИВШЕМСЯ ПРОЦЕССЕ ПРОКАТКИ
}

Совершенствование существующих и создание новых прогрессивных машин и технологий обработки металлов требует от проектантов, разработчиков и исследователей всестороннего, системного подхода к изучаемому объекту. Прикладной системный анализ основан на рассмотрении объекта деятельности как технической системы, предназначенной для выполнения заданных функций и взаимодействия с внешней средой. Способность технических систем к развитию и самоорганизации $[1,2]$ открывает возможности находить эффективные пути совершенствования техники и технологии.

В соответствии с принятой парадигмой валки и полосу, взаимодействующие в очаге деформации и через межклетевые промежутки, следует рассматривать как развивающуюся динамическую систему, в которой нужно поддерживать устойчивость и стабильность (допустимая область), обеспечивая непопадание процесса прокатки в область неустойчивости контролем параметров-критериев [2].

При изменении параметров прокатки или при других внешних воздействиях, выводящих из допустимой области, в очаге деформации возникает нарушение равновесия сил, что приводит к частичной или полной пробуксовке металла в валках, т.е. к неконтролируемому изменению состояния динамической системы. Эти условия определяют границу устойчивого состояния системы. В теории она оценивается равенством:

$$
\gamma=0
$$

где $\gamma$ - угол нейтрального сечения.

Вместе с тем экспериментальные данные А. П. Чекмарева [3] и других исследователей [4] показывают, что равновесие сил в очаге деформации может нарушаться с потерей устойчивости системы и при значительном опережении $(\gamma>0)$ ). Заметим, что с выражением (1) тесно связан предельный угол захвата в установившемся режиме $\alpha_{y}^{\max }=2 f_{y}$ [2].

Применим системный подход при анализе процесса прокатки в предельных условиях. Согласно исследованиям Хейла и других $[5,6]$ в этих условиях по всей длине очага деформации, за исключением границ, возникают продольные нормальные растягивающие напряжения, и эпюра распределения давления имеет вид вогнутой кривой. При этом среднее давление $p_{\mathrm{cp}}<2 k_{\mathrm{cp}}\left(2 k_{\mathrm{cp}}-\right.$ среднее сопротивление деформации). С учетом потерь на трение при простом процессе прокатки это неравенство невозможно по энергетическим соображениям. Следовательно, потеря равновесия полосы в валках с последующей пробуксовкой должна произойти при меньшем угле захвата. Поэтому граничное условие $\gamma=0$ требует уточнения.

Изменения параметров процесса прокатки при его совершенствовании будут допустимыми, если не изменяется состояние системы-технологии, оцениваемое как устойчивое: раскат деформируется в клетях прокатного стана в нормативном режиме, вариации параметров не приводят к потере устойчивости процесса.

Цель работы заключается в оценке продольной устойчивости полосы при прокатке с помощью адекватного критерия, а также в уточнении граничных условий процесса в установившемся режиме.

Следуя принципу системности, на основе исследования контактных и продольных нормальных напряжений, а также текущих горизонтальных сил, продолжим анализ предель- 
ных условий прокатки в установившемся режиме. Для этого, по численным решениям уравнения Т. Кармана при удельных силах трения, заданных законом Кулона, построены эпюры распределения $p_{x}$ для трех случаев прокатки, при которых изменяется коэффициент $f_{y}=0,06 ; 0,041 ; 0,03$. В расчетах принято, что угол $\alpha_{y}=0,06$ рад, начальная толщина полосы $h_{0}=1$ мм, а радиус валков $R=100$ мм. Результаты расчетов приведены на рис. 1 . Они качественно совпадают с данными исследований $[8,9]$.
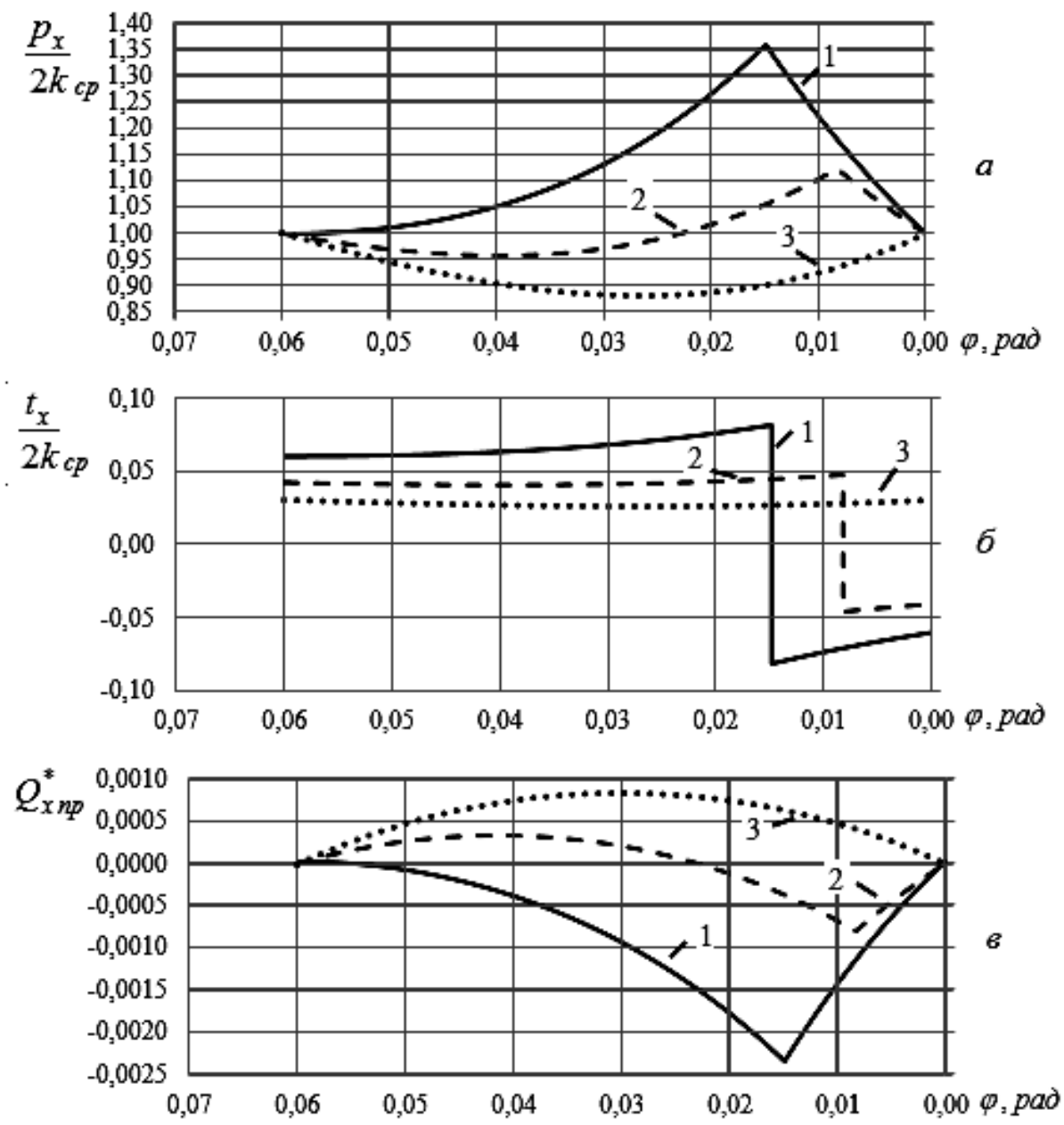

Рис. 1. Относительные контактные напряжения $(a, \sigma)$ и результирующая продольных сил (в) в очаге деформации

Как видно при $\frac{\alpha_{y}}{f_{y}}=1$ (кривые 1) давление в каждом сечении очага деформации заметно превышает среднее сопротивление металла деформации.

Продольные нормальные напряжения $\sigma_{x}$ являются сжимающими

$$
\sigma_{X}=p_{X}-2 k_{c p} \text {. }
$$

При этом внутренние текущие продольные силы определяются по формуле:

$$
Q_{x n p}=\sigma_{X} h_{x} b_{x}
$$

или в безразмерной форме

$$
Q_{x n p}^{*}=\frac{Q_{x n p}}{2 k_{c p} h_{x} R}\left(\frac{h_{1}}{R}+\varphi^{2}\right)
$$


и во всех сечениях напрвлены противоположно по отношению к движению полосы (рис. 2).

Аналогично направлена и средняя результирующая этих сил

$$
Q_{c p n p}^{*}=\frac{1}{\alpha_{y}} \int_{0}^{\alpha_{y}} Q_{x n p}^{*} d \varphi
$$

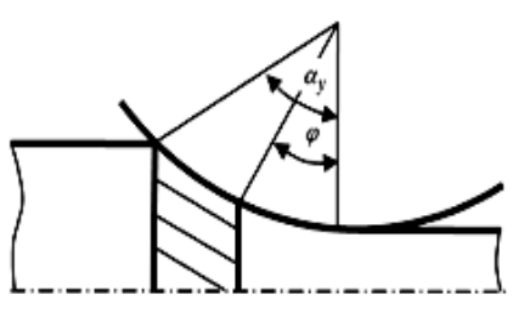

$a$

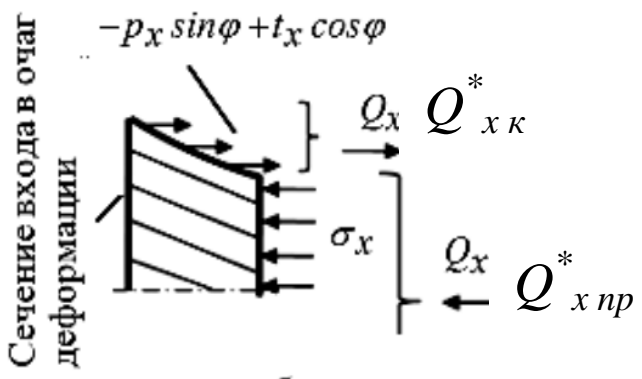

$\sigma$

Рис. 2. К равновесию горизонтальных сил:

$a$ - очаг деформации; $\sigma$ - выделенный текущий объем металла

В случае прокатки, когда $\alpha_{y}=2 f_{y}$ (рис. 1, кривые 3) по всей длине очага деформации действуют продольные напряжения растяжения, текущие силы $Q_{x n p}^{*}$ становятся активными, направленными в соответствии с движением полосы, как бы помогая процессу прокатки. В этих условиях и средняя результирующая $Q_{c p n p}^{*}$ также выступает в роли активной силы. Но ведь эта внутренняя сила пластически деформируемого металла является силой сопротивления и поэтому активную (движущую) роль выполнять не может. Следовательно, в указанных условиях процесс устойчиво совершаться не может. Приведенные соображения поясняют, почему в условиях $\alpha_{y}=2 f_{y}$ прокатка невозможна.

Проанализируем характер изменения $Q_{x}^{*}$ в случае, когда $\frac{\alpha_{y}}{f_{y}}=\frac{0,06}{0,042}=1,429$ (рис. 1 , кривая 2). Как следует из эпюры на одной части очага деформации продольные внутренние силы, являясь сжимающими силами, направлены противоположно ходу прокатки, на другой они совпадают с направлением движения полосы и являются растягивающими. Характер движения полосы и продольная устойчивость процесса прокатки зависит от величины средней результирующей продольной силы $Q_{c p}^{*}$. Площади участков разнонаправленных продольных внутренних сил $Q_{x}^{*}$ в в данном случае одинаковы, суммарный результат нулевой. Тогда уравнение

$$
Q_{c p n p}^{*}=0
$$

определяет предельное условие для установившегося процесса прокатки.

Неизменность направления действия средней результирующей силы $Q_{c p \text { пр }}^{*}$ можно также объяснить следующими соображениями. Для этого рассмотрим равновесие сил, действующих на выделенный текущий объем металла, который находится в очаге деформации (рис. 2):

$$
-2 \int_{\varphi}^{\alpha_{y}} \frac{p_{x}}{2 k_{c p}} \sin \varphi d \varphi+2 \int_{\varphi}^{\alpha_{y}} \frac{t_{x}}{2 k_{c p}} \cos \varphi d \varphi=\frac{\sigma_{x}}{2 k_{c p}}\left(\frac{h_{1}}{R}+\varphi^{2}\right) .
$$


Как известно, левая часть уравнения (6) представляет собой текущую горизонтальную проекцию контактных сил в безразмерной форме, обозначим ее через $Q_{x \kappa}^{*}$. Тогда уравнение (6) можно представить в виде:

$$
Q_{x k}^{*}=Q_{x n p}^{*} .
$$

Аналогичная связь будет и между среднеинтегральными значениями этих сил:

$$
Q_{c p k}^{*}=Q_{c p n p}^{*},
$$

где $Q_{c p k}^{*}=\frac{1}{\alpha_{y}} \int_{0}^{\alpha_{y}} Q_{x k}^{*} d \varphi$.

Векторы сил, величины которых входят в уравнение (8), противонаправлены. Сила $Q_{c p \kappa}^{*}$ является активной, она действует со стороны валков на металл. Внутренняя сила $Q_{c p ~ n p}^{*}$, как уже указывалось, является силой сопротивления. Проанализируем характер взаимодействия между этими силами. Пусть по каким-либо причинам средняя результирующая внутренних сил изменила свое направление, например, из-за существенного увеличения продольных нормальных растягивающих напряжений. Тогда в соответствии с (8) должна изменить свое направление и сила $Q_{c p}^{*}$. В этом случае втягивающих металл в валки сил будет недостаточно для обеспечения процесса прокатки и произойдет торможение полосы в валках. Следовательно, и при совместном анализе средних контактных и внутренних сил в очаге деформации предельное условие прокатки в установившемся режиме должно характеризо-

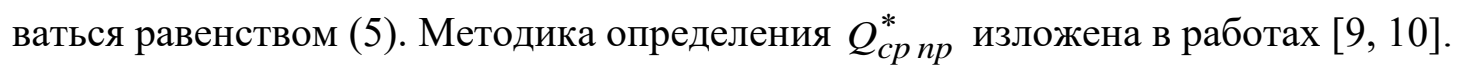

Необходимо заметить, что предельное условие (5) может наступать и при наличии опережения (см. кривую 2, рис. 1), что подтверждается результатами опытов [3, 6]. Проведенные расчеты показали, что отношение предельного угла захвата к коэффициенту трения при использовании условия $Q_{c p n p}=0$ находится в промежутке $\frac{\alpha_{y}}{f_{y}} \in[1,42 ; 1,55]$. Это отношение слабо зависит от толщины полосы после прохода и диаметра валков. Анализ показывает, что отношение $\frac{\alpha_{y}^{n p e d}}{f_{y}}$, рассчитанное по условию (5), дает значения заметно меньшие в сравнении с известным в теории прокатки предельным условием. Следует подчеркнуть, что предлагаемое предельное условие может быть использовано при оценке продольной устойчивости полосы в валках при прокатке с натяжением [10,11].

В качестве примера приведено исследование продольной устойчивости процесса при прокатке листа на непрерывном четырехклетевом стане 1680 комбината «Запорожсталь». При существующих режимах деформации и натяжения полосы [12] результаты расчетов средней результирующей силы $Q_{c p n p}^{* \mu}$ представлены в табл. 1.

В связи с тем, что средняя внутренняя сила $Q_{c p n p}^{* H}$ при нормальном процессе прокатки направлена противоположно движению полосы припишем ей знак «минус». Как следует из расчетных значений этой силы при существующем режиме натяжения, прокатка во всех клетях совершается устойчиво без нарушения условий равновесия полосы в валках. Следует заметить, что во второй и третьей клетях прокатка ведется при пониженной стабильности. Авторы работы [12] с целью снижения энергозатрат при прокатке предложили увеличить натяжения между клетями (табл. 2). 
Как видно, в этом случае, средняя результирующая сила $Q_{c p n p}^{* H}$ в очагах деформации второй и третьей клети меняет свой знак (направление действия), что должно привести к неустойчивому поведению полосы в валках и к пробуксовке металла.

Таблица 1

Параметры режимов обработки и результаты расчета продольной устойчивости полосы при прокатке листа $0,8 \times 1000$ мм из подката 2,5 × 1000 мм на четырехклетевом стане 1680

\begin{tabular}{|c|c|c|c|c|}
\hline № клети & 1 & 2 & 3 & 4 \\
\hline$h_{1}$, мм & 1,65 & 1,22 & 0,9 & 0,8 \\
\hline \multicolumn{5}{|c|}{ Существующий режим натяжения } \\
\hline$p_{c p}, H /$ мм $^{2}$ & 637 & 580 & 623 & 715 \\
\hline$\alpha$, рад & 0,0556 & 0,0343 & 0,028 & 0,0117 \\
\hline$f_{y}$ & 0,113 & 0,0819 & 0,0703 & 0,081 \\
\hline$\sigma_{0} / \sigma_{T 0}$ & 0,159 & 0,355 & 0,351 & 0,363 \\
\hline$\sigma_{1} / \sigma_{T 1}$ & 0,355 & 0,351 & 0,363 & 0,062 \\
\hline$Q_{c p n p}^{* H}$ & $-0,0032$ & $-0,00001$ & $-0,000091$ & $-0,00038$ \\
\hline
\end{tabular}

Примечание: $p_{c p}$ - среднее давление в очаге деформации; $\sigma_{0}$ - заднее удельное натяжение полосы; $\sigma_{1}$ - переднее удельное натяжение полосы;

$\sigma_{T 0}, \sigma_{T 1}-$ предел текучести металла на входе и выходе из клети;

$Q_{c p n p}^{* \mu}-$ безразмерная средняя результирующая сила с учетом натяжения полосы.

Таблица 2

Характер изменения силы $Q_{c p n p}^{* H}$ при увеличении натяжения металла между клетями

\begin{tabular}{|c|c|c|c|c|}
\hline$p_{c p}, H /$ мM $^{2}$ & 626 & 551 & 582 & 681 \\
\hline$\alpha, p a \partial$ & 0,0558 & 0,0346 & 0,0286 & 0,0119 \\
\hline$f_{y}$ & 0,115 & 0,082 & 0,0703 & 0,0815 \\
\hline$\sigma_{0} / \sigma_{T 0}$ & 0,159 & 0,39 & 0,403 & 0,423 \\
\hline$\sigma_{1} / \sigma_{T 1}$ & 0,39 & 0,403 & 0,423 & 0,062 \\
\hline$Q_{c p n p}^{* H}$ & $-0,0033$ & 0,00022 & 0,000135 & $-0,00029$ \\
\hline
\end{tabular}

Следовательно, увеличение натяжения между клетями, с одной стороны, влияет на снижение суммарных энергозатрат, с другой - отражается на стабильности процесса непрерывной прокатки. Поэтому при корректировке технологических режимов рекомендуется учитывать предельное условие устойчивой прокатки расчетом значений удельной средней результирующей силы, которые должны оставаться отрицательными.

\section{ВЫВОДЫ}

На основе системного подхода с учетом контактных напряжений и внутренних продольных сил пластически деформируемого металла получено новое предельное условие прокатки в установившемся режиме, которое является более адекватным опытным и производственным данным, чем известные критерии в теории прокатки. Рекомендуется использовать это граничное условие при корректировке технологических параметров прокатки в энергосберегающих процессах обработки. 


\section{СПИСОК ИСПОЛЬЗОВАННОЙ ЛИТЕРАТУРЫ}

1. Згуровский М. 3., Панкратова Н. Д. Системный анализ. Проблемы, методология, приложения: монография. Киев: Наукова думка. 2011. 727 с.

2. Максименко О. П., Никулин А. В., Лобойко Д. И. Системный аспект продольной устойчивости процесса прокатки. Збірник наукових праць Дніпровського державного технічного університету (технічні науки). Кам'янське : ДДТУ. 2019. 2(35). С. 16-23.

3. Чекмарев А. П., Прокофьев В. И., Галицкий В. П. и др. Экспериментальное исследование максимальных углов захвата при установившемся процессе прокатки. Обработка металлов давлением: Науч. труды, ДМетИ. Москва: Металлургия. 1967. 52. С. 79-88.

4. Грудев А. П. Теория прокатки. 2-е изд. перераб. и доп. Москва: СП Интермет Инжиниринг. 2001.280 с.

5. Хейн А. Я. Процесс ленточной и тонколистовой прокатки. Москва: Металлургиздат. 1941. 247 с. $1998.283 \mathrm{c}$

6. Грудев А. П. Захватывающая способность прокатных валков. Москва: СП Интермет Инжиниринг.

7. Целиков А. И., Гришков А. И. Теория прокатки. Москва: Металлургия. 1970. 358 с.

8. Максименко О. П., Лобойко Д. И., Измайлова М. К. Продольная устойчивость полосы в валках с анализом контактных условий: монография. Днепродзержинск: ДГТУ. 2016. 213 с.

9. Максименко О. П., Романюк Р. Я. Методика оценки продольной устойчивости процесса прокатки. Известия ВУЗов. Черная металлургия. 2009. 10. С. 22-24.

10. Максименко О. П., Измайлова М. К., Лобойко Д. И. Продольная устойчивость процесса прокатки с натяжением полосы при двухзвенной модели трения в очаге деформации. Металлургическая и горнорудная промышленность. 2015. 4. С. 73-76.

11. Максименко О. П., Лобойко Д. И., Васильев А. А., Присяжный А. Г. Расчет рационального режима натяжения полосы с учетом ее продольной устойчивости в очаге деформации. Металлургическая и горнорудная промышленность. 2018. 2. С. 16-20.

12. Василев Я. Д., Самокиш Д. И. Разработка энергосберегающих режимов натяжения на непрерывных станах холодной прокатки. Металлургическая и горнорудная промышленность. 2013. 2. С. 34-38.

\section{REFERENCES}

1. Zgurovskiy M.Z., Pankratova N.D. System analysis. Problems, methodology, applications: monograph. Kyiv : Scientific thought. 2011. 727 p. (in Russian).

2. Maksimenko O.P., Nikulin A.V., Loboyko D.I. Systemic aspect of longitudinal stability of the rolling process. Collection of scientific works of the Dnieper State Technical University (technical sciences). Kamyanske : DSTU. 2019. 2(35), pp. 16-23. (in Russian).

3. Chekmarev A.P., Prokofev V.I., Galitskiy V.P. et al. Experimental study of maximal capture angles during steady rolling process. Metal Forming: Sci. works, DMetI. Moscow : Metallurgy. 1967. 52, pp. 79-88. (in Russian).

4. Grudev A.P. Rolling theory. Moscow : JV Intermet Engineering. 2001. 280 p. (in Russian).

5. Heyn A.Ya. Strip and sheet rolling process. Moscow : Metallurgizdat. 1941. 247 p. (in Russian).

6. Grudev A.P. Exciting the ability of the rolls. Moscow : JV Intermet Engineering. 1998. 283 p. (in Russian).

7. Tselikov A.I., Grishkov A.I. Rolling theory. Moscow: Metallurgy. 1970. 358 p. (in Russian).

8. Maksimenko O.P., Loboyko D.I., Izmaylova M.K. Longitudinal stability of strip in rolls with analysis of contact conditions: monograph. Dniprodzerzhinsk: DSTU. 2016. 213 p. (in Russian).

9. Maksimenko O.P., Romanyuk R.Ya. Method for assessing the longitudinal stability of the rolling process. Izvestiya. Ferrous metallurgy. 2009. 10, pp. 22-24. (in Russian).

10. Maksimenko O.P., Izmaylova M.K., Loboyko D.I. Longitudinal stability of rolling process with strip tension in a two-link model of friction in the deformation zone. Metallurgical and mining industry. 2015. 4, pp. 73-76. (in Russian).

11. Maksimenko O.P., Loboyko D.I., Vasilev A.A., Prisyazhnyiy A.G. Calculation of the rational mode of strip tension taking into account its longitudinal stability in the deformation zone. Metallurgical and mining industry. 2018. 2, pp. 16-20. (in Russian).

12. Vasilev Ya.D., Samokish D.I. Development of energy-saving tensioning modes on continuous cold rolling mills. Metallurgical and mining industry. 2013. 2, pp. 34-38. (in Russian).

Максименко О. П. - - д-р техн. наук, проф. ДГТУ; E-mail: O.maks1940@gmail.com

Никулин А. В. $\quad$ - канд. техн. наук, доц. ДГТУ; E-mail: av_nikulin@ukr.net

Лобойко Д. И. $\quad$ - канд. техн. наук, ДГТУ. E-mail: darloboyko@gmail.com

ДГТУ - Днепровский государственный технический университет, г. Каменское. 